\title{
Supporting Information \\ Logarithmic data processing can be used justifiably in plotting of calibration curve
}

Dezhao Kong ${ }^{\mathrm{a}}$, Jun Zhao ${ }^{\mathrm{b}}$, Sheng Tang ${ }^{\mathrm{c}, *}$, Wei Shen ${ }^{\mathrm{c}}$, Hian Kee Lee ${ }^{\mathrm{d}, *}$

aSchool of Grain Science and Technology, Jiangsu University of Science and Technology, Zhenjiang, People's Republic of China;

'School of Science, Jiangsu University of Science and Technology, Zhenjiang, People's Republic of China;

'School of Environment and Chemical Engineering, Jiangsu University of Science and Technology, Zhenjiang, People's Republic of China;

dDepartment of Chemistry, National University of Singapore, 3 Science Drive 3, Singapore, 117543, Singapore

Corresponding authors' e-mail addresses: tangsheng.nju@gmail.com (S. Tang);

chmleehk@nus.edu.sg (H.K. Lee) 


\section{Table of Contents}

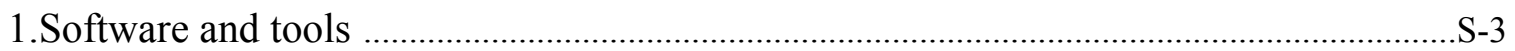

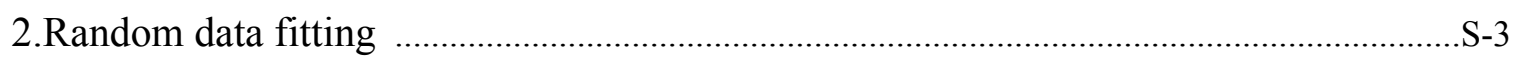

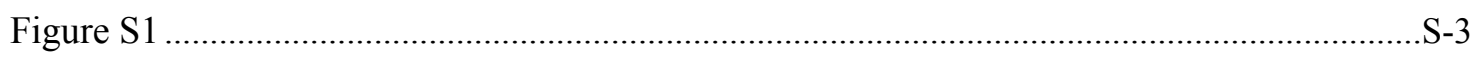

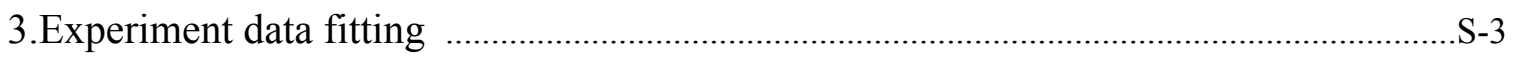

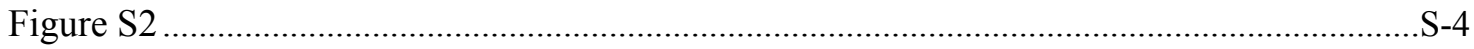




\section{Software and tools}

All data analysis was performed using Python software. The sklearn.linear_model is used for linear fitting, and the scipy.optimize.curve_fit is used for nonlinear fitting. The main toolkits in Python chooses for data analysis are numpy, pandas, scipy, and sklearn, etc.

\section{Random data fitting}

A simple function as $\mathrm{Y}=1.3+2.5 \log \mathrm{X}$ is selected for verification of data deviation. Random data is generated by the numpy kit in Python based on the chosen function, and then used for function fitting with abscissa in linear and logarithmic, respectively (Figure S1). The $\mathrm{R}^{2}$ value (coefficient of determination, used to represent the fitting degree of regression line fits the observed value) and RMSE value (root-mean-square error, used to measure the deviation between the observed value and the true value) are calculated.

For nonlinear-regression model fitting with linear abscissa, the obtained $\mathrm{R}^{2}$ is 0.999 , and the RMSE value is 0.236 . For linear-regression model fitting with abscissa of the logarithm, the obtained $\mathrm{R}^{2}$ is 0.999 , and the RMSE value is 0.254 . The transformation of the axes will not affect the coefficient of determination value in the two fitting functions. The approximate RMSE value proves that the average deviation of each point is nearly consistent with the point on the corresponding position curve.
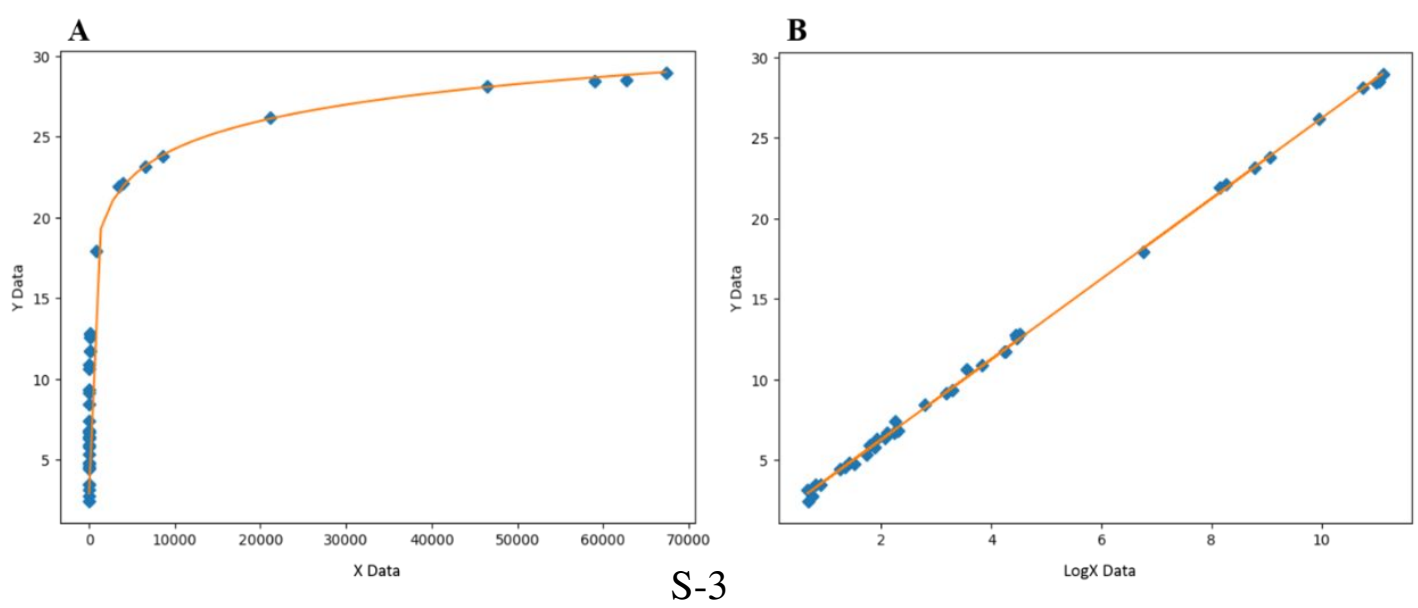
Figure S1. Random data fitting result, (A) fitting result of nonlinear-regression model, (B) fitting result of linear-regression model.

\section{Experiment data fitting}

Except for software-generated data, complete and partial experimental data were used for data analysis by Python, respectively. And the $\mathrm{R}^{2}$ value and RMSE value are calculated. (Figure S2)

For nonlinear-regression model fitting with linear abscissa of complete experimental data, the obtained $\mathrm{R}^{2}$ is 0.937 , and the RMSE value is 0.178 (Figure S2 A). For linear-regression model fitting with abscissa of the logarithm, the obtained $\mathrm{R}^{2}$ is 0.928 , and the RMSE value is 0.268 (Figure S2 B). In complete experimental data analysis, both fitting methods will lead to an unsatisfactorily low $\mathrm{R}^{2}$ value because of the large range of the abscissa. Also, the RMSE value will show a certain difference. For nonlinear-regression model fitting with linear abscissa of partial experimental data, the obtained $\mathrm{R}^{2}$ is 0.998 , and the RMSE value is 0.270 (Figure S2 C). For linear-regression model fitting with abscissa of the logarithm, the obtained $\mathrm{R}^{2}$ is 0.997 , and the RMSE value is 0.270 (Figure S2 D). Appropriate abscissa range selection will lead to a better $\mathrm{R}^{2}$ value and similar RMSE for each fitting method.
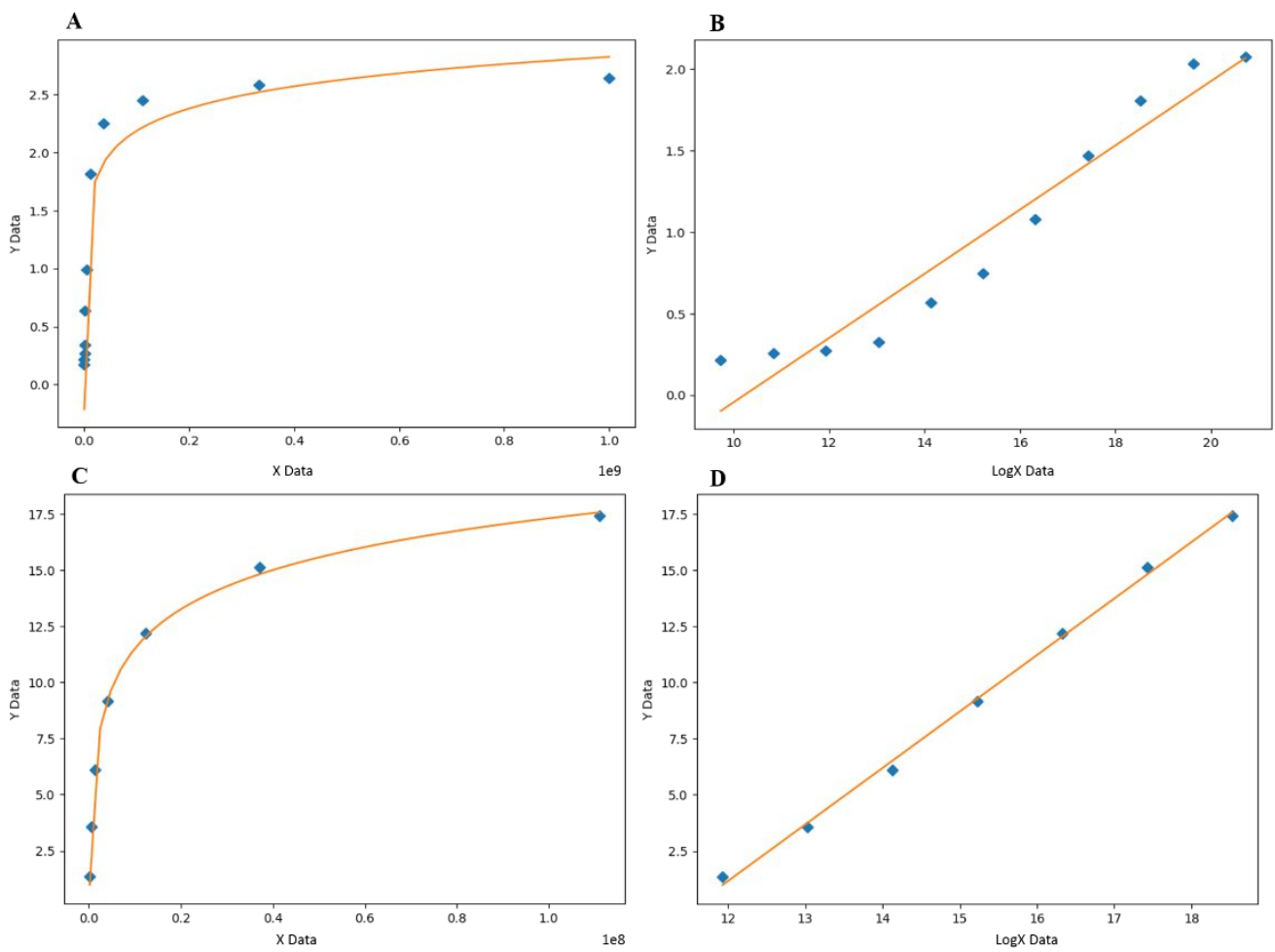
Figure S2. Experiment data fitting result, (A) fitting result of nonlinear-regression model with complete experimental data, (B) fitting result of linear-regression model with complete experimental data, (C) fitting result of nonlinear-regression model with partial experimental data, (D) fitting result of linear-regression model with partial experimental data.

In software analysis, compare with use concentration data as abscissa directly, enabling logarithmic processing of concentration data as abscissa will not significantly affect the parameters of the fitting function, such as the $\mathrm{R}^{2}$ and RMSE values. Therefore, these different model transformation methods can be interchanged, and it is reasonable to use the logarithmic data as abscissae for function fitting. 